\title{
MHC genetic diversity and avian malaria prevalence in Mokoia Island saddlebacks
}

\author{
Jolene T. Sutton ${ }^{1,2,3^{*}}$, Isabel Castro ${ }^{4}$, Bruce C. Robertson ${ }^{1,2}$, Daniel M. Tompkins ${ }^{5}$ \\ Jo-Ann L. Stanton ${ }^{6}$ and Ian G. Jamieson ${ }^{1,2, \dagger}$ \\ ${ }^{1}$ Allan Wilson Centre for Molecular Ecology and Evolution \\ 2 Department of Zoology, University of Otago, PO Box 56, Dunedin 9054, New Zealand \\ ${ }^{3}$ Department of Biology, University of Hawai'i at Hilo, Hilo, USA \\ ${ }^{4}$ Institute of Agriculture and the Environment, Massey University, Private Bag 11 222, Palmerston North 4442, New Zealand \\ ${ }^{5}$ Landcare Research, Private Bag 1930, Dunedin 9054, New Zealand \\ ${ }^{6}$ Department of Anatomy, University of Otago, Dunedin, New Zealand \\ ${ }^{\dagger}$ Died 2 February 2015 \\ *Author for correspondence (Email: jtsutton@hawaii.edu)
}

Published online: 13 May 2016

\begin{abstract}
Studies of wildlife populations have identified associations between disease resistance and diversity at genes of the major histocompatibility complex (MHC), which are involved with adaptive immunity. We compare MHC class II B (MHCIIB) and microsatellite genetic diversity in a population of New Zealand passerine birds, North Island saddlebacks on Mokoia Island, that was also tested for avian malaria. Prevalence of infection was low, and we found no conclusive evidence that infection status was linked to particular MHCIIB alleles. Individual-level MHCIIB nucleotide diversity, and the number of alleles per bird were both lower in infected compared with uninfected samples; however, neither trend was statistically significant. Genetic structure was observed in the MHCIIB dataset, suggesting that selective forces are shaping MHCIIB diversity in this population. Future research would benefit from long-term monitoring of allele frequencies and larger sample sizes where possible. Additionally, including disease intensity data (i.e. the degree of infection in an individual) alongside prevalence data (i.e. presence/absence of infection in an individual) may enhance the ability to detect phenotype-genotype associations in future research.
\end{abstract}

Keywords: bottleneck; infectious disease; mosquito-vectored; next generation sequencing; (Philesturnus rufusater; Plasmodium)

\section{Introduction}

Small populations with reduced genetic diversity may be highly susceptible to infection by novel pathogens. Although still equivocal, there is growing evidence that wild populations with low diversity at genes of the major histocompatibility complex (MHC) may be especially compromised (e.g. Siddle et al.2007, reviewed in Radwan et al.2010). MHC genes initiate adaptive immune responses by encoding cell-surface proteins that are responsible for recognising and binding to foreign peptides (Klein \& O’hUigin 1993). Class I MHC molecules are primarily associated with intracellular pathogens, such as viruses, while class II molecules target peptides derived mainly from extracellular pathogens (Hughes \& Yeager 1998; Piertney \& Oliver 2006). Evidence from wild populations indicates that disease associations can occur for specific class I and class II alleles, as well as for groups of alleles and/or overall MHC diversity (e.g. Hill et al. 1991; de Campos-Lima et al. 1993; Thursz et al. 1995; Paterson et al. 1998; Trachtenberg et al. 2003; Wegner et al. 2003; Westerdahl et al. 2005; Bonneaud et al. 2006; Siddle et al. 2007; Oliver et al. 2009; Jarvi et al. 2013; Sepil et al. 2013). Additionally, while negative associations with disease may be interpreted as certain $\mathrm{MHC}$ genotypes providing qualitative protection against a pathogen, positive associations may result from quantitatively resistant genotypes that limit pathogen load and/or the deleterious effects of the disease, without completely eradicating it (Sepil et al. 2013). The latter phenomenon is also referred to as 'tolerance'. Characterising MHC-disease associations may be particularly useful for species of conservation concern, as such information may help guide management actions. For example, eastern and northwestern populations of Tasmanian devils (Sarcophilus harrisii) have different MHC profiles, and maintaining isolation between these populations has been proposed for managing outbreaks of an infectious cancer (Siddle et al. 2010). In New Zealand, genetic information has increasingly been incorporated into wildlife management strategies, particularly for avian species, and there is mounting effort to understand and incorporate patterns of immune gene diversity (e.g. Jamieson 2015).

Many endemic New Zealand bird species are threatened due to population bottlenecks over the last century that have also led to losses of genetic diversity, including reduced MHC diversity (Holdaway 1989; Miller \& Lambert 2004; Jamieson et al. 2008; Sutton et al. 2013; Jamieson 2015; Sutton et al. 2015). It has been speculated that this reduced genetic diversity may increase the vulnerability of these species to disease (Hale 2007; Hale \& Briskie 2007). One concern is the potential susceptibility of New Zealand birds to introduced strains of avian malaria. Avian malaria is a mosquito-transmitted disease caused by protozoan parasites of the genus Plasmodium (Order Haemosporida). These parasites asexually multiply in bird red blood cells (Valkiūnas et al. 2005; Braga et al. 2011), and undergo sexual reproduction in mosquitoes (Diptera: Culicidae). In infected birds, pathological effects can include altered pigmentation of the blood, anaemia, 
morbidity, and mortality (Braga et al. 2011). The first exposure to avian malaria usually results in an acute infection phase with the most severe fitness consequences (Atkinson \& van Riper 1991), after which the infection may persist for years with little or no quantifiable effects on fitness (i.e. chronic infection; Atkinson \& van Riper 1991). Naïve species with no prior exposure to malaria can be particularly susceptible to the disease, leading to dramatic losses of avifauna following spread of the parasites and their mosquito vectors (van Riper III et al. 1986; Atkinson \& van Riper 1991). In Hawai'i, for example, introduced avian malaria and its introduced mosquito vector, Culex quinquefasciatus, play a key role in the ongoing declines and extinctions of many endemic forest bird species (van Riper III et al. 1986; Atkinson et al. 2014).

Unlike Hawaiian species, New Zealand native birds may have had an association with Plasmodium spp. that pre-dates human colonisation (i.e. before 1000 years ago; Baillie \& Brunton 2011; Ewen et al. 2012; Howe et al. 2012; Schoener et al. 2014). There are at least two endemic Plasmodium lineages (Ewen et al. 2012), and of the twelve native mosquito species, at least one, C. pervigilans, is a confirmed vector (Derraik 2004; Massey et al. 2007). Additionally, non-native Plasmodium have been co-introduced with exotic birds, likely since the 1800s (Duncan et al. 2006; Ewen et al. 2012), and one introduced species, $P$. relictum, can be highly virulent in some avian hosts (Atkinson et al. 1995; Palinauskas et al. 2008). Range expansion over the past 35 years by the exotic mosquito C. quinquefasciatus, which is a competent vector of $P$. relictum, has prompted concern that negative impacts on native avifauna could mirror those seen elsewhere in the world (Tompkins \& Gleeson 2006). To date, avian malaria has been reported in several native New Zealand species (reviewed in Derraik et al. 2008, Howe et al. 2012, and Schoener et al. 2014), and has been implicated in the deaths of mōhua (Mohoua ochrocephala), New Zealand dotterel (Charadrius obscurus; Alley 2002; Derraik et al. 2008), South Island saddleback (Philesturnus carunculatus; Alley et al. 2010), great spotted kiwi (Apteryx haastii; Howe et al. 2012), and hihi (Notiomystis cincta; Howe et al. 2012).

Recently, New Zealand North Island saddlebacks ( $P h$. rufusater: Calleidae) on Mokoia Island were discovered to be infected with multiple lineages of Plasmodium at low prevalence ( $6.3 \%$ of 319 sampled birds), and it is believed that this population harbours chronic infections (Thorne 2007; Castro et al. 2011). Previous research has shown that North Island saddlebacks have moderately variable MHC class II diversity (Sutton et al. 2013, 2015), but to the best of our knowledge no published studies have aimed to link avian malaria susceptibility to MHC variation in any native New Zealand species. Elsewhere, studies have identified associations between malaria and diversity at MHC class I and class II genes (e.g. Westerdahl et al. (2005) for associations with class I genes; Sepil et al. (2013) for associations with class II genes; Hill et al. (1991) for associations with both classes to human malaria). Here, we investigate whether avian malaria infection is associated with MHC class II B (hereafter, MHCIIB) genetic diversity in the Mokoia Island North Island saddleback population. We predict that if specific MHCIIB alleles convey resistance/susceptibility to avian malaria in North Island saddlebacks, then infected and uninfected birds will have different MHCIIB profiles (i.e. some alleles will occur at high frequencies in one group, but will be absent or at low frequency in the other). Additionally, as individuals with greater MHCIIB diversity may be able to respond to a wider array of pathogens, possibly including a wider range of Plasmodium lineages, uninfected birds may have greater MHCIIB diversity. For comparison with MHCIIB results, we use microsatellite markers to test whether patterns at $\mathrm{MHC}$ genes deviate from those expected under neutrality.

\section{Methods}

\section{Sample collection, disease testing \& genotyping}

Blood samples from 319 Mokoia Island saddlebacks were collected between January 2007 and March 2008 (i.e. spring/ summer/autumn; concurrent with the saddleback breeding season), and screened for Plasmodium spp. infection as described in Castro et al. (2011). Twenty (6.3\%) birds were positive from a PCR-based test (Castro et al. 2011). To acquire MHCIIB and microsatellite profiles for subsets of uninfected and infected birds, we first used a modified chelex method (Walsh et al. 1991; Casquet et al. 2012) to extract genomic DNA from blood stored in lysis buffer (Seutin et al. 1991). For our MHCIIB analysis, we performed sample preparation, sequencing, and bioinformatics based on the protocol outlined in Sutton et al. $(2013,2015)$ for 14 uninfected and 16 infected birds. Note that because samples for this project were sequenced alongside those for concurrent studies, we had to limit our sample sizes so that we could maximise sequencing coverage per individual. To ensure very good coverage, we chose to sequence a total of 30 saddlebacks from Mokoia Island, 16 of which came from the infected pool of samples, and 14 from the uninfected pool. Briefly, our protocol involved amplifying each sample in triplicate with barcoded primers PhcaMHCII.F.1 and PhcaMHCII.Ex2.R.1 (Sutton et al. 2013) to target 240 bp ( $~ 90 \%)$ of exon 2 , followed by pooling and purifying (Agencourt ${ }^{\circledR}$ AMPure XP, Beckman Coulter) the amplified products from each individual. We then quantified each purified product (Qubit ${ }^{\mathrm{TM}}$, Invitrogen ${ }^{\mathrm{TM}}$ ), and pooled equimolar amounts of all samples for next generation sequencing (Ion Torrent ${ }^{\mathrm{TM}}$, Life Technologies (Rothberg et al. 2011)). We filtered sequences by length and quality, and removed low frequency sequencing reads $(<0.001$ of variants $)$. We retained sequences that were $314-320 \mathrm{bp}$, which represented the expected amplicon size (with barcoded primers) \pm 3 bp to allow for \pm 1 codon differences. Our quality restrictions retained sequences for which $\geq 90 \%$ of nucleotides had Phred scores of at least 15 . The per-base quality (Phred) scores obtained from the sequencing files are defined by the expression $q=$ $-10 \times \log (p)$, where $p$ is the probability of error (Ewing \& Green 1998; Ewing et al. 1998). Thus, a score of 15 indicates $96.8 \%$ accuracy. Finally, we assigned sequences to individual birds based on unique barcode combinations (see Sutton et al. 2013, 2015 for complete bioinformatics protocol). Although individual sequences could not be assigned to specific loci due to the nature in which passerine MHC loci are typically coamplified(e.g. Babik etal. 2009; Zagalska-Neubauer etal. 2010; Sepil et al. 2012), we hereafter refer to individual sequences as 'alleles' for simplicity. We estimated the repeatability of our methods for validating and assigning alleles to individuals by comparing the alleles assigned to replicate samples from 23 saddlebacks included in this and concurrent studies. We calculated our repeatability scores as 1-APD (average percent difference; Yuhki \& O’Brien 1990).

For our microsatellite analysis, we used multiplex PCR to amplify 23 loci (Table S1 in online Supplementary Material) in all 20 infected and a subset of 20 uninfected birds. We 
dried 10-15 ng of DNA into wells of 384-well plates before PCR. Each $2 \mu \mathrm{l}$ reaction contained $1 \mu \mathrm{l}$ Type-it Master Mix (Qiagen), and $1 \mu \mathrm{l}$ primer mix containing forward (0.02-0.44 $\mu \mathrm{M})$ and reverse $(0.08-1.76 \mu \mathrm{M})$ locus-specific primers in addition to M13-tagged (Schuelke 2000) fluorescent 'dyes' (6-FAM, VIC, NED, PET; $0.16 \mu \mathrm{M} \times$ number of loci). We were consistent with our assignment of dye to locus in order to avoid genotyping error due to dye-associated allele size shifts (Sutton et al. 2011). Our cycling conditions were $95^{\circ} \mathrm{C}$ for $5 \mathrm{~min}$, followed by a touchdown sequence comprising $94^{\circ} \mathrm{C}$ for $30 \mathrm{~s}$, annealing for $90 \mathrm{~s}$ and extension at $72^{\circ} \mathrm{C}$ for $45 \mathrm{~s}$. During the touchdown cycles, the annealing temperature started at $60^{\circ} \mathrm{C}$ and reduced 1 degree per cycle for 8 cycles. This was followed by 25 cycles at $94^{\circ} \mathrm{C}$ for $30 \mathrm{~s}, 52^{\circ} \mathrm{C}$ for $90 \mathrm{~s}$ and $72^{\circ} \mathrm{C}$ for $45 \mathrm{~s}$ with a final $30 \mathrm{~min}$ hold at $60^{\circ} \mathrm{C}$. Following amplification, we resolved fragment sizes on an ABI 3730 Genetic Analyser (Applied Biosystems; Genetic Analysis Services, University of Otago) with GeneScan 500 $(\mathrm{LIZ})^{\mathrm{TM}}$ size standard, and used Genemapper v4.0 (Applied Biosystems) to score alleles. To check data for null alleles and scoring errors, we used Micro-checker v2.2.3 (van Oosterhout et al. 2004). Deviations from Hardy-Weinberg equilibrium (HWE) were calculated in Genepop v4.0.10 (Raymond \& Rousset 1995; Rousset 2008), using the exact probability test (Guo \& Thompson 1992) with Markov chain parameters employing 10000 dememorisations, 1000 batches and 10000 iterations per batch. To test for linkage disequilibrium, we used Genpop probability tests (Fisher's exact tests) with the same Markov chain parameters used for testing HWE. We applied sequential Bonferroni correction (Holm 1979; Rice 1989) to the resulting $\mathrm{p}$-values.

\section{MHC comparisons}

When considering multi-locus MHCIIB profiles (i.e. all the MHCIIB alleles identified as present in an individual), only four genotypes occurred more than once (one occurred twice, two occurred three times, and one occurred eight times; the genotype that occurred twice, and the genotype that occurred eight times were found in uninfected and infected individuals). Therefore, to investigate differences in MHCIIB profiles between uninfected and infected birds, we focused on testing for associations between infection status and the presence or absence of each allele independently. We used Fisher's exact tests implemented in R v3.1.2 (R Core Team 2014) to assess whether the presence of each allele was significantly different between uninfected and infected birds. As sample sizes for MHC genotypes were small and slightly different for uninfected and infected birds (14 vs16), we also used R to randomly assign individuals to each infection status without replacement 10000 times (i.e. permutations). We used these permutations to generate a predicted mean and $95 \%$ confidence interval of the presence of each allele given a null hypothesis that presence was not associated with infection status.

We used in-house $\mathrm{R}$ code to generate an individual fasta file of the alleles present in each bird so that we could calculate individual-level statistics for the total number of alleles $\left(A_{i}\right)$, the total number of polymorphic sites $\left(S_{i}\right.$; using the R function 'seg. sites' in package ape; (Paradis et al. 2004)), and the nucleotide diversity $\left(\pi_{i}\right.$; using the $\mathrm{R}$ function 'nuc.div' in package pegas (Paradis 2010)). We used generalised linear models (GLMs, implemented in R) to compare estimates between infected and uninfected birds. Finally, we tested for genetic structure (i.e. patterns of genetic relationships) by using Genalex v6.41 (Peakall \& Smouse 2006) to first calculate genetic distance between uninfected and infected samples, and then to perform Principal Components Analysis (PCA) for visualising the patterns of genetic structure contained in the distance matrix. Distance matrices can be calculated for binary as well as codominant genetic markers, so to estimate genetic distance for our MHCIIB data we treated each allele as either present (1) or absent (0) in each sample, and we selected the distance calculation option for binary (haploid) data.

In addition to treating alleles as being distinct in terms of their effect on phenotype, we also tested for the effects of MHC 'functional supertypes' on infection status. Functional supertypes consist of groups of alleles predicted to have similar antigen-binding properties based on their amino acid sequences (e.g. Trachtenberg et al. 2003; Sepil et al. 2013). We considered five MHCIIB functional supertypes that were previously identified for North Island saddlebacks (Sutton 2013), based on the amino acid properties of each individual allele ( $z$-descriptors; Sandberg et al. 1998; Doytchinova $\&$ Flower 2005; Schwensow et al. 2007), and by $k$-means clustering using the adegenet package in R (Jombart 2008). To test whether the number of supertypes per individual was different between uninfected and infected birds, we used GLMs with binomial distributions implemented in $\mathrm{R}$.

\section{Microsatellite comparisons}

To investigate differences in microsatellite profiles between uninfected and infected birds, we used Genalex to calculate expected and observed heterozygosities (respectively, $H_{E}$ and $H_{O}$ (Nei 1978)) for each group. We used Fstat v2.9.3.2 (Goudet 1995,2002 ) to calculate allelic richness (number of alleles per population corrected for sample size (Mousadik \& Petit 1996; Petit et al. 1998)), and we assessed genetic differentiation in Arlequin v3.5 (Excoffier et al. 2005) by using Slatkin's linearised $F_{\mathrm{ST}}$ (Slatkin 1995). Significance of $F_{\mathrm{ST}}$ values was tested using 30000 permutations. As with our MHCIIB data, we used Fisher's exact tests to assess whether the presence of each allele was significantly different between uninfected and infected birds. To visualise genetic structure, we implemented PCA and assignment tests in Genalex.

\section{Results}

\section{MHC}

Sequencing results

A total of 69498 reads were assigned to this study based on exact matches of barcodes on both the forward and reverse primers. The average number of reads per individual was 2316 ( \pm 628 SD, range $975-3685$ ), with $>0.95$ estimated repeatability of our genotyping methods. Our data yielded a total of 15 putatively functional MHCIIB alleles (Genbank accessions: KF225751-KF225754; KF225756-KF225763; KF225765KF225766; KP125357) that did not contain stop codons, and which displayed additional characteristics of classical MHC alleles (e.g. they did not cluster with a group of non-classical MHCIIB sequences that were previously identified; Sutton et al. 2013). All alleles were $240 \mathrm{bp}$, and differed from each other by at least 5 nucleotides (2.1\%). Each allele translated a unique amino acid sequence.

\section{MHC genetic diversity}

All 15 MHCIIB alleles were present in the sample of infected birds, while 14 were observed in the uninfected birds (Fig. 1). 
Most alleles were observed in relatively equal proportions between the two groups of samples, and the one allele that was missing from the uninfected birds (PhruMHCII001) was only present in $19 \%$ of infected birds (Fig. 1). The allele that had the greatest disparity between groups was PhruMHC027, which occurred in $36 \%$ of uninfected birds and $75 \%$ of infected birds (Fig. 1, Table 1). However, the presence of individual MHCIIB alleles was not significantly different between the two groups (Fisher's exact tests, Table 1). The number of copies of individual alleles fell within the $95 \%$ confidence interval from 10000 permutations for each infection status, further demonstrating that allele presence was not significantly associated with infection status.

When we compared individual-level variation in terms of the number of alleles per bird $\left(A_{i}\right)$, the number polymorphic sites $\left(S_{i}\right)$, and nucleotide diversity $\left(\pi_{i}\right)$, we observed nonsignificant trends of lower diversity in infected birds compared to uninfected birds (Table $2 \&$ Fig. 2). Of the five MHC supertypes identified previously (Sutton 2013), all five were found in both uninfected and infected birds. Although infected birds had, on average, fewer supertypes per individual (mean \pm standard deviation: uninfected $=4.86 \pm 0.36$; infected $=4.13$ \pm 1.15 ), this difference was not significant (GLM: z-value $=$ $1.778 \mathrm{p}$-value $=0.075$ )

MHC genetic structure

Plotting the first two axes of the PCA, which was based on pairwise genetic differences, showed that individuals could be divided into two groups (Fig. 3a). These first two axes explained $73 \%$ of the MHCIIB variation in the 30 Mokoia Island saddleback samples. Nineteen birds clustered into a 'low diversity' group to the left along axis 1, while eleven birds clustered into a 'high diversity' group on the right (Fig. $3 a)$. Of the 15 total MHCIIB alleles, $40 \%$ were missing from the low diversity group, while none were absent from the high diversity group. Clustering did not appear to be associated with infection status (Fig. 3a).

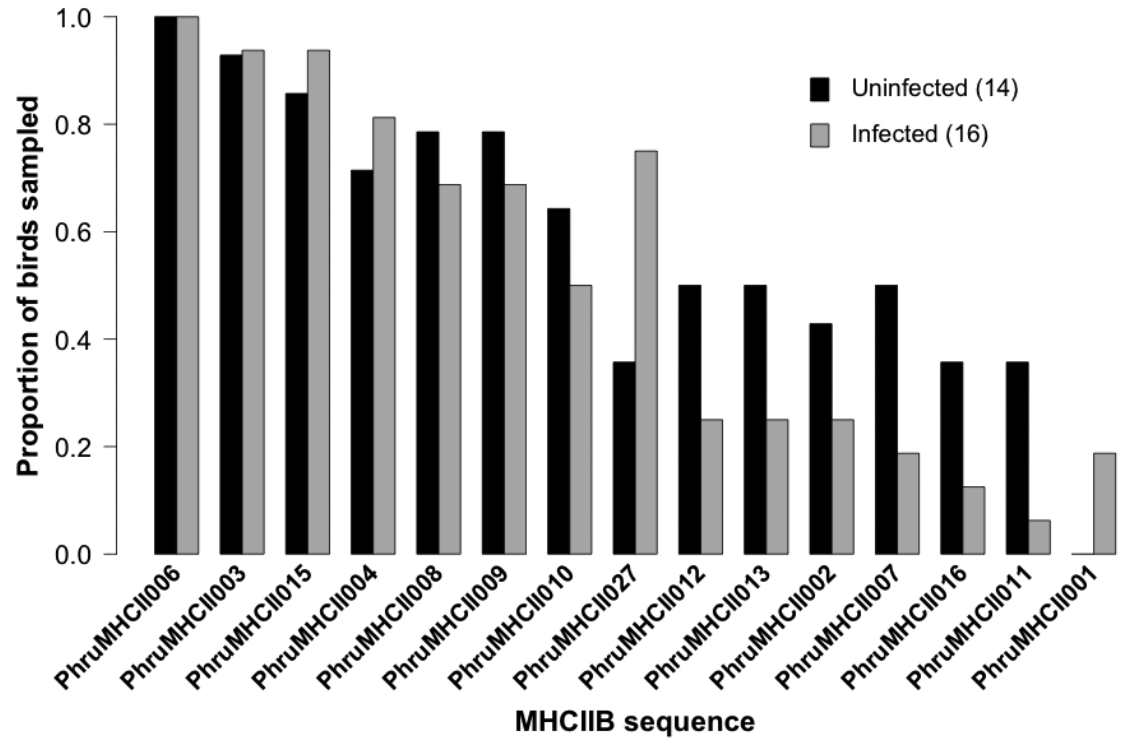

Figure 1: Identities of MHCIIB sequences and proportions at which each was present in Mokoia Island saddlebacks infected and uninfected with avian malaria. Legend numbers indicate sample sizes.

Table 1: Results of Fisher's exact tests comparing the presence of each MHCIIB allele in Mokoia Island saddlebacks infected and uninfected with avian malaria.

\begin{tabular}{|c|c|c|c|c|c|c|c|}
\hline Allele & $\mathbf{N}$ & $\begin{array}{l}\text { Number of } \\
\text { uninfected } \\
\text { birds (max } \\
N=14)\end{array}$ & $\begin{array}{l}\text { Number of } \\
\text { infected } \\
\text { birds (max } \\
\mathrm{N}=16)\end{array}$ & $\begin{array}{l}\text { Fisher's } \\
\text { odds ratio }\end{array}$ & $\begin{array}{l}\text { Fisher's } \\
\text { p-value }\end{array}$ & $\begin{array}{l}\text { Permutation } \\
\text { mean in } \\
\text { uninfected } \\
\text { birds }(95 \% \mathrm{CI})\end{array}$ & $\begin{array}{l}\text { Permutation } \\
\text { mean in } \\
\text { infected birds } \\
(95 \% \mathrm{CI})\end{array}$ \\
\hline PhruMHC001 & 30 & 0 & 3 & 0.000 & 0.228 & $1.4(0-3)$ & $1.6(0-3)$ \\
\hline PhruMHC002 & 30 & 6 & 4 & 2.188 & 0.442 & $4.7(2-7)$ & $5.3(3-8)$ \\
\hline PhruMHC003 & 30 & 13 & 15 & 0.871 & 1.000 & $13.1(12-14)$ & $14.9(14-16)$ \\
\hline PhruMHC004 & 30 & 10 & 13 & 0.588 & 0.675 & $10.7(8-13)$ & $12.3(10-15)$ \\
\hline PhruMHC006 & 30 & 14 & 16 & na & na & $16(16-16)$ & $14(14-14)$ \\
\hline PhruMHC007 & 30 & 7 & 3 & 4.111 & 0.122 & $4.7(2-7)$ & $5.3(3-8)$ \\
\hline PhruMHC008 & 30 & 11 & 11 & 1.639 & 0.689 & $10.3(8-13)$ & $11.7(9-14)$ \\
\hline PhruMHC009 & 30 & 11 & 11 & 1.639 & 0.689 & $10.3(8-13)$ & $11.7(9-14)$ \\
\hline PhruMHC010 & 30 & 9 & 8 & 1.765 & 0.484 & $7.9(5-11)$ & $9.1(6-12)$ \\
\hline PhruMHC011 & 30 & 5 & 1 & 7.771 & 0.072 & $2.8(1-5)$ & $3.2(1-5)$ \\
\hline PhruMHC012 & 30 & 7 & 4 & 2.887 & 0.257 & $5.1(3-8)$ & $5.9(3-8)$ \\
\hline PhruMHC013 & 30 & 7 & 4 & 2.887 & 0.257 & $5.1(3-8)$ & $5.9(3-8)$ \\
\hline PhruMHC015 & 30 & 12 & 15 & 0.412 & 0.586 & $12.6(11-14)$ & $14.4(13-16)$ \\
\hline PhruMHC016 & 30 & 5 & 2 & 3.712 & 0.204 & $3.3(1-5)$ & $3.7(2-6)$ \\
\hline PhruMHC027 & 30 & 5 & 12 & 0.197 & 0.063 & $7.9(5-11)$ & $9.1(6-12)$ \\
\hline
\end{tabular}


Table 2: Individual-level MHCIIB exon 2 sequence polymorphism in Mokoia Island saddlebacks infected and uninfected with avian malaria based on the average number of sequences per bird $\left(A_{i}\right)$, the average number polymorphic sites (segregating, $\left.S_{i}\right)$ among sequences, and nucleotide diversity $\left(\pi_{i}\right)$. Numbers in brackets indicate standard deviations. $Z$ - and p-values come from GLMs with binomial distributions, for which degrees of freedom were 29 (total) and 28 (residual) for all models.

\begin{tabular}{lrrrr}
\hline $\begin{array}{l}\text { MHC } \\
\text { diversity }\end{array}$ & Uninfected & Infected & z-value & p-value \\
\hline$A_{i}$ & $8.71(2.43)$ & $7.63(2.19)$ & 1.259 & 0.208 \\
$S_{i}$ & $78.43(6.41)$ & $76.06(7.07)$ & 0.962 & 0.336 \\
$\pi_{i}$ & $0.133(0.004)$ & $0.131(0.002)$ & 1.576 & 0.115 \\
\hline
\end{tabular}

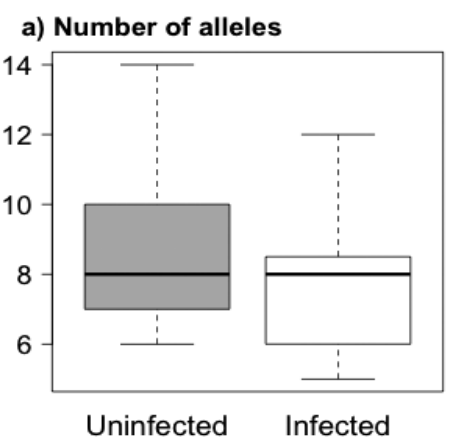

(14)

(16)

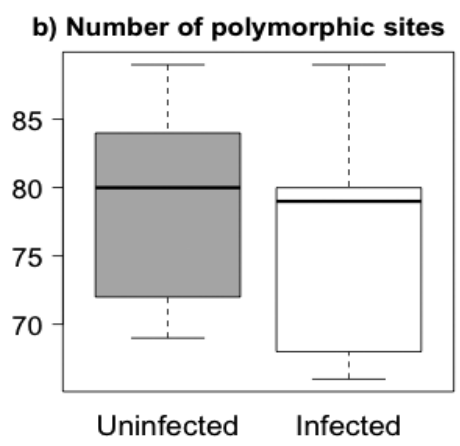

(14)

(16)

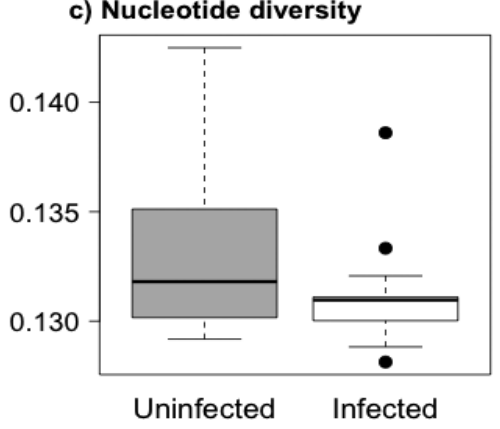

(14)

(16)

Figure 2: Individual-level MHCIIB exon 2 polymorphism in Mokoia Island saddlebacks infected and uninfected with avian malaria Horizontal bars represent median values. Boxes above and below median values indicate the interquartile range (the $25^{\text {th }}$ to $75^{\text {th }}$ percentile). Where there are no outliers, the whiskers represent the maximum and minimum values. Where there are outliers (filled circles), the whiskers represent 1.5 times the interquartile range (approximately two standard deviations). Values in brackets along the $\mathrm{x}$-axis indicate sample sizes.
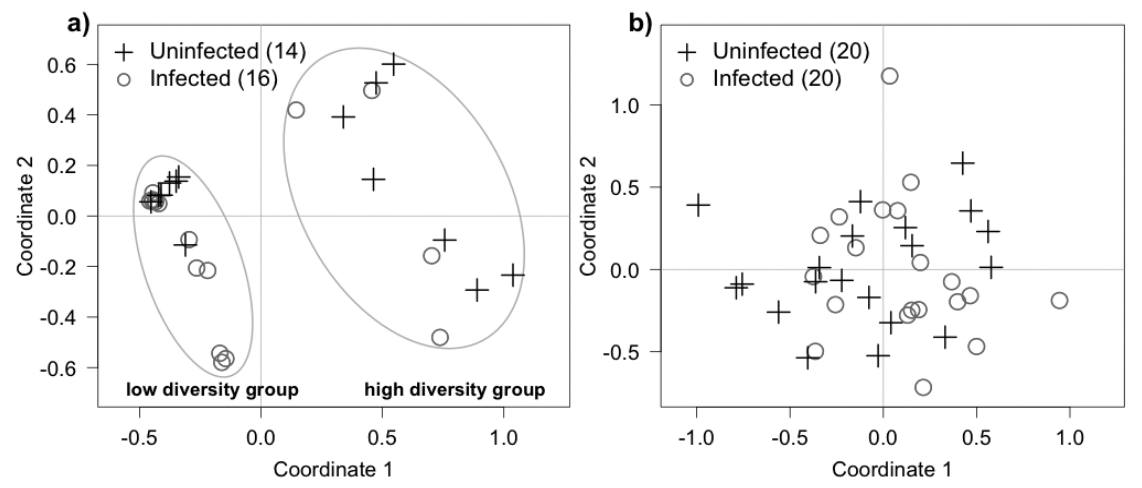

Figure 3: Plot of the first two axes of a PCA based on presence/absence of a) 15 MHCIIB sequences, and b) microsatellite genotype $(n$ loci $=19)$ in Mokoia Island saddlebacks infected and uninfected with avian malaria. Data-points are jittered to reduce overlap. The two axes in panel (a) explain $73 \%$ of the total variation. The two axes in panel (b) explain $44 \%$ of the total variation.

\section{Microsatellites}

Tests for Hardy-Weinberg departures and genotyping errors Of the 23 microsatellite loci that we amplified, 19 were polymorphic in Mokoia Island saddlebacks (loci CK5A4B, K3/K4, PCC02 and POCC6 (Table S1) were monomorphic). Among the 19 polymorphic loci, there were no deviations from Hardy-Weinberg proportions, and no evidence of null alleles or linkage disequilibria. All samples amplified at least 17 loci, and $93 \%$ of samples amplified all 19 polymorphic loci. We estimated genotyping error rate using data from a concurrent study involving 486 samples, which included 40 Mokoia Island samples. Across the 19 loci, the proportion of samples that were re-genotyped ranged from $15.5 \%$ to $42.3 \%$ per locus $($ mean $=23.8 \%)$, and resulted in an overall error rate of $0.27 \%$.

Microsatellite genetic diversity

In total, 47 alleles were recorded across 19 microsatellite loci in 40 Mokoia Island saddleback samples. Allelic richness standardised to sample size $(n=19)$ was 2.36 for uninfected and infected birds. There was no difference between mean $H_{O}$ and mean $H_{E}$ within each group, and mean $H_{O}$ was similar between uninfected and infected birds (mean $H_{O} \pm$ standard error: uninfected $=0.392 \pm 0.048$; infected $=0.381 \pm 0.050$ ). 


\section{Microsatellite genetic structure}

There were two private microsatellite alleles (i.e. alleles that only occur in one group) in the uninfected birds (one each at loci $2 \mathrm{~F} 9$ and $2 \mathrm{H} 8 / \mathrm{Pca} 05)$ and two private alleles in the infected birds (one each at loci 4H2/Pca14 and Pcc42). Each private allele was rare, occurring at a frequency of 0.025 . For comparison with MHC data, this frequency corresponded to $5 \%$ of either infected or uninfected individuals. Fisher's exact tests indicated that the presence of each allele did not differ significantly between uninfected and infected birds. The pairwise $F_{\mathrm{ST}}$ was zero, indicating no differentiation between infected and uninfected birds based on their microsatellite genotypes $(p$-value $=0.614)$. A two-dimensional PCA plot (Fig. 3b) showed no clustering, and the variation explained by the first two axes was $44 \%$. Population assignment tests correctly assigned only $48 \%$ of samples according to their infection status.

\section{Discussion}

We examined MHC and microsatellite genetic diversity in a population of New Zealand North Island saddlebacks that was exposed to avian malaria on Mokoia Island. Genes of the MHC are associated with immunity, while diversity at microsatellite loci is assumed to be neutrally evolving. Thus, comparing patterns of MHC and microsatellite diversity is a useful strategy for testing for selection, and assessing phenotype-genotype associations. We found no significant difference in the presence of individual MHCIIB alleles between infected and uninfected birds. However, we note that MHC allele PhruMHC001 was only found in infected birds (19\% of infected individuals), and MHC allele PhruMHC027 occurred in a substantially larger proportion of infected than uninfected birds (75\% vs 36\%). By comparison, differences between microsatellite alleles were much smaller. As our sample sizes were necessarily low due to low prevalence of avian malaria at the time of this study, it would be worthwhile monitoring the frequencies of $\mathrm{MHC}$ alleles over time in North Island saddlebacks, particularly PhruMHC001 and PhruMHC027, to further assess the possibility of a phenotype-genotype association. For example, if there is a weak phenotype-genotype association (e.g. if an allele has a small effect on tolerance/susceptibility to avian malaria), then the frequency of that allele in the population should change slowly rather than quickly over time. This could explain the moderately large, but non-significant, disparity between the presence of PhruMHC027 in uninfected and infected birds at the time of sampling. Alternatively, if birds with a particular allele succumb quickly to avian malaria during the acute infection stage, then that allele would be difficult to detect in wild populations where sick or dead individuals may be not sampled (Westerdahl et al. 2005).

In terms of overall genetic diversity and structure, we observed a non-significant trend toward higher individual-level MHCIIB polymorphism in uninfected birds. Further, there was evidence of genetic structuring in MHCIIB genotypes, which did not occur for neutrally evolving microsatellite loci. Although a difference in genetic structuring between $\mathrm{MHC}$ and microsatellite diversity is suggestive of balancing selection acting on the MHC (Piertney \& Oliver 2006; Alcaide 2010), we did not find conclusive evidence that MHC genetic structuring was associated with avian malaria infection status in the Mokoia Island saddleback population. Thus, we cannot identify specific factors that may be responsible for MHC genetic structuring. We also cannot discount the possibility that there may be an association between MHC diversity and intensity of infection, which we were unable to test at this time.

Assuming that phenotype-genotype associations exist, then a failure to detect associations could result from a number of factors aside from small sample sizes. As described above, highly susceptible individuals might be omitted during sampling if they are less active than healthy individuals, or if they die from their infections (Westerdhal et al. 2005). Second, as native New Zealand birds are probably not naïve to some avian malaria parasites or their mosquito vectors, it is possible that this co-evolutionary history imparts a measure of resilience against introduced lineages. In this scenario, historical selection pressures could have led to contemporary birds having similar, resistant genotypes. At least two Plasmodium lineages (BELL01 and KOKAK001) are thought to be endemic to New Zealand (Baillie \& Brunton 2011; Ewen et al. 2012; Howe et al. 2012), and a native New Zealand mosquito, C. pervigilans, is a confirmed Plasmodium vector (Massey et al. 2007). Thus, birds that become infected with new strains of Plasmodium may not necessarily suffer severe infection. Third, most non-native Plasmodium species were likely co-introduced to New Zealand during colonial times (Ewen et al. 2012). Thus, even if a history of co-evolution with one Plasmodium lineage did not bestow resistance to a novel strain, enough time may have passed for some New Zealand species/populations to have experienced evolution in response to non-native Plasmodium, similar to what appears to be occurring in low elevation populations of the Hawaiian amakihi (Hemignathus virens) after introductions of $P$. relictum to the Hawaiian Islands in the early 1800 s (Foster et al. 2007).

Contrary to the idea that a co-evolutionary history with native Plasmodium imparts a degree of resilience to introduced lineages, or that enough time has passed for resistance to have evolved, infection by new or multiple strains, or in combination with other parasites, may raise parasitaemia levels beyond the capability of the host's immune system. At least two of the Plasmodium lineages identified in North Island saddlebacks from Mokoia Island phylogenetically resemble P. relictum, but other lineages, including the native KOKAK001, were also found (Castro et al. 2011; Howe et al. 2012). One lineage that was commonly identified in saddlebacks on Mokoia Island was P. (Huffia) elongatum (Castro et al. 2011), which has also been identified in other species, including the carcasses of South Island saddlebacks co-infected with avian pox (Alley et al. 2010; Howe et al. 2012). Although it may be difficult to determine the primary causative agent following diseaserelated mortality, future research may benefit by comparing MHC profiles in birds that succumb vs birds that survive disease outbreaks. For example, comparisons of MHC profiles following experimental infections of avian malaria have identified specific groups of alleles associated with either survival or susceptibility in Hawaiian honeycreepers (Jarvi et al. 2013).

Spread of non-native avian malaria parasites and their mosquito vectors can have catastrophic consequences when naïve hosts become infected. In Hawai' $i$, introductions of $P$. relictum and the mosquito vector $C$. quinquefasciatus have decimated several endemic bird populations, and continue to impede conservation efforts (Atkinson et al. 1995, 2000, 2014). On a global scale, associations between avian malaria resistance/susceptibility and the $\mathrm{MHC}$ have been identified in several species (e.g. Westerdahl et al. 2005, 2012; Bonneaud et al. 2006; Loiseau et al. 2008; Radwan et al. 2012; Dunn et 
al. 2013; Jarvi et al. 2013; Sepil et al. 2013). Excluding studies in which birds were experimentally infected, one factor that these cited studies have in common is that malaria prevalence was high $(\geq 30 \%)$ in nearly all populations studied, and ranged from $33.3 \%$ to $81.6 \%$ (mean $=52.2 \%$; standard deviation $=$ 14.2) in populations for which associations between MHC variation and infection probability were identified. Thus, higher prevalence than that observed in the Mokoia Island saddleback population may increase power to detect correlations between MHC variation and infection status. Additionally, more extensive datasets that include parasite intensity could help elucidate complex host-disease dynamics, which are likely to be context specific, and correlated with such factors as host age and reproductive status, food availability, and environmental variation (Knowles et al. 2011). Saddleback populations that have higher prevalence (e.g. Hen Island, E.R. Schoener, New Zealand, pers. comm.), as well as other New Zealand species with higher prevalence (e.g. bellbird (Anthornis melanura) populations in the Hauraki Gulf (Baillie et al. 2012)) are candidates for such research, and would make excellent comparisons for low prevalence populations like Mokoia Island saddlebacks.

\section{Acknowledgements}

We thank the team of volunteers who collected blood samples, as well as Laryssa Howe for performing PCR screening for Plasmodium. Laura Martin, Beth Ross, and Emily Weiser provided valuable comments to earlier manuscript drafts. Isabel Castro was funded by grants from Massey University Research Fund, the Department of Conservation and Biosecurity NZ contract 10424/2 (March 2008), and by Landcare Research in-kind contributions. Sampling was carried out under Massey University Animal Ethics Approval 06/111; permit 2006/85 NZ Banding Office; and written permission from the Mokoia Island Māori Trust Board. This research was also funded by grants to Ian Jamieson from Landcare Research, University of Otago, and Allan Wilson Centre for Molecular Ecology and Evolution. Jolene Sutton was supported by scholarships from Natural Sciences and Engineering Research Council of Canada (NSERC) and New Zealand Ministry of Education(NZIDRS).

\section{References}

Alcaide M 2010. On the relative roles of selection and genetic drift in shaping MHC variation. Molecular Ecology 19: 3842-3844.

Alley M2002. Avian wildlife diseases in New Zealand: current issues and achievements. New Zealand Veterinary Journal 50: $118-120$.

Alley M, Hale K, Cash W, Ha H, Howe L 2010. Concurrent avian malaria and avipox virus infection in translocated South Island saddlebacks (Philesturnus carunculatus carunculatus). New Zealand Veterinary Journal 58: 218-223.

Atkinson CT, van Riper C 1991. Pathogenicity and epizootiology of avian haematozoa: Plasmodium, Leucocytozoon and Haemoproteus. In: Loye JE, Zuk Meds Bird-parasite interactions. New York, Oxford University Press. Pp. 19-48.

Atkinson C, Woods K, Dusek R, Sileo L, Iko W 1995. Wildlife disease and conservation in Hawaii: pathogenicity of avian malaria (Plasmodium relictum) in experimentally infected Iiwi (Vestiaria coccinea). Parasitology 111: 59-S69.

Atkinson CT, Dusek RJ, Woods KL, Iko WM 2000. Pathogenicity of avian malaria in experimentally-infected HawaiiAmakihi. Journal of Wildlife Diseases 36: 197-204.

Atkinson CT, Utzurrum RB, Lapointe DA, Camp RJ, Crampton LH, Foster JT, Giambelluca TW 2014. Changing climate and the altitudinal range of avian malaria in the Hawaiian Islands - an ongoing conservation crisis on the island of Kaua'i. Global Change Biology 20: 2426-2436.

Babik W, Taberlet P, Ejsmond MJ, Radwan J 2009. New generation sequencers as a tool for genotyping of highly polymorphic multilocus MHC system. Molecular Ecology Resources 9: 713-719.

Baillie S, Brunton D 2011. Diversity, distribution and biogeographical origins of Plasmodium parasites from the New Zealand bellbird (Anthornis melanura). Parasitology 138: 1843-1851.

Baillie SM, Gudex-Cross D, Barraclough RK, Blanchard W, Brunton DH 2012. Patterns in avian malaria at founder and source populations of an endemic New Zealand passerine. Parasitology Research 111: 2077-2089.

Bonneaud C, Perez-Tris J, Federici P, Chastel O, Sorci G 2006. Major histocompatibility alleles associated with local resistance to malaria in a passerine. Evolution 60:383-389.

Braga ÉM, Silveira P, Belo NO, Valkiknas G 2011. Recent advances in the study of avian malaria: an overview with an emphasis on the distribution of Plasmodium spp in Brazil. Memorias do Instituto Oswaldo Cruz 106: 3-11.

Casquet J, Thebaud C, Gillespie RG 2012. Chelex without boiling, a rapid and easy technique to obtain stable amplifiable DNA from small amounts of ethanol-stored spiders. Molecular Ecology Resources 12: 136-141.

Castro I, Howe L, Tompkins DM, Barraclough RK, Slaney D 2011. Presence and seasonal prevalence of Plasmodium spp. in a rare endemic New Zealand passerine (tieke or saddleback, Philesturnus carunculatus). Journal of Wildlife Diseases 47: 860-867.

de Campos-Lima P-O, Gavioli R, Zhang Q-J, Wallace LE, Dolcetti R, Rowe M, Rickinson AB, Masucci MG 1993. HLA-A11 epitope loss isolates of Epstein-Barr virus from a highly A11+ population. Science 260: 98-100.

Derraik JG 2004. Exotic mosquitoes in New Zealand: a review of species intercepted, their pathways and ports of entry. Australian and New Zealand Journal of Public Health 28: 433-444.

Derraik JGB, Tompkins DM, Alley MR, Holder P, Atkinson $\mathrm{T}$ 2008. Epidemiology of an avian malaria outbreak in a native bird species (Mohoua ochrocephala) in New Zealand. Journal of the Royal Society of New Zealand 38: 237-242.

Doytchinova IA, Flower DR 2005. In silico identification of supertypes for class II MHCs. The Journal of Immunology 174: 7085-7095.

Duncan RP, Blackburn TM, Cassey P 2006. Factors affecting the release, establishment and spread of introduced birds in New Zealand. Biological Invasions in New Zealand: 137-154.

Dunn PO, Bollmer JL, Freeeman-Gallant CR, Whittingham LA 2013. MHC variation is related to a sexually selected ornament, survival, and parasite resistance in common yellowthroats. Evolution 67: 679-687.

Ewen JG, Bensch S, Blackburn TM, Bonneaud C, Brown R, Cassey P, Clarke RH, Pérez-Tris J 2012. Establishment 
of exotic parasites: the origins and characteristics of an avian malaria community in an isolated island avifauna. Ecology Letters 15: 1112-1119.

Ewing B, Green P 1998. Base-calling of automated sequencer traces using phred. II. Error probabilities. Genome research 8: 186-194.

Ewing B, Hillier L, Wendl MC, Green P 1998. Base-calling of automated sequencer traces using Phred. I. Accuracy assessment. Genome research 8: 175-185.

Excoffier L, Laval G, 2005. Arlequin ver. 3.0: An integrated software package for population genetics data analysis. Evolutionary Bioinformatics Online 1: 47-50.

Foster JT, Woodworth BL, Eggert LE, Hart PJ, Palmer D, Duffy DC, Fleischer RC 2007. Genetic structure and evolved malaria resistance in Hawaiian honeycreepers. Molecular Ecology 16: 4738-4746.

Goudet J 1995. FSTAT, a program to estimate and test gene diversities and fixation indices (version 1.2). Journal of Heredity 86: 485-486.

Goudet J 2002. FSTAT version 2.9.3.2. University of Lausanne, Lausanne, Switzerland.

Guo S, Thompson E 1992. Performing the exact test of HardyWeinberg proportion for multiple alleles. Biometrics 48: 361-372.

Hale KA 2007. Population bottlenecks and the risk of parasitic and microbiological infections. PhD Thesis, University of Canterbury, Christchurch, New Zealand.

Hale KA, Briskie JV 2007. Decreased immunocompetence in a severely bottlenecked population of an endemic New Zealand bird. Animal Conservation 10: 2-10.

Hill AV, Allsopp CE, Kwiatkowski D, Anstey NM, Twumasi P, Rowe PA, Bennett S, Brewster D, McMichael AJ, Greenwood BM 1991. Common West African HLA antigens are associated with protection from severe malaria. Nature 352: 595-600.

Holdaway R 1989. New Zealand's pre-human avifauna and its vulnerability. New Zealand Journal of Ecology 12: 11-25.

Holm S 1979. A simple sequentially rejective multiple test procedure. Scandinavian Journal of Statistics: 65-70.

Howe L, Castro I, Schoener E, Hunter S, Barraclough R, Alley M 2012. Malaria parasites (Plasmodium spp.) infecting introduced, native and endemic New Zealand birds. Parasitology Research 110: 913-923.

Hughes A, Yeager M 1998. Natural selection at the major histocompatibility complex loci of vertebrates. Annual Review of Genetics 32: 415-435.

Jamieson IG 2015. Significance of population genetics for managing small natural and reintroduced populations in New Zealand. New Zealand Journal of Ecology 39:1-18.

Jamieson IG, Grueber CE, Waters JM, Gleeson DM 2008. Managing genetic diversity in threatened populations: a New Zealand perspective. New Zealand Journal of Ecology 32: 130-137.

Jarvi S, Farias M, Txakeeyang A, McFarland T, Atkinson C 2013. Next generation sequencing reveals major histocompatibility complex class II alleles associated with survival to avian malaria (Plasmodium relictum) in Hawaiian honeycreepers (Drepanidinae)(P3089). The Journal of Immunology 190: 125.19.

Jombart T 2008. adegenet: a R package for the multivariate analysis of genetic markers. Bioinformatics 24: 1403-1405.

Klein J, O'hUigin C 1993. Composite origin of major histocompatibility complex genes. Current Opinion in Genetics \& Development 3: 923-930.
Knowles SCL, Wood MJ, Alves R, Wilkin TA, Bensch S, Sheldon BC 2011. Molecular epidemiology of malaria prevalence and parasitaemia in a wild bird population. Molecular Ecology 20: 1062-1076.

Loiseau C, Zoorob R, Garnier S, Birard J, Federici P, Julliard R, Sorci G 2008. Antagonistic effects of a Mhc class I allele on malaria-infected house sparrows. Ecology Letters 11: 258-265.

Massey B, Gleeson D, Slaney D, Tompkins D 2007. PCR detection of Plasmodium and blood meal identification in a native New Zealand mosquito. Journal of Vector Ecology 32: 154-156.

Miller HC, Lambert DM 2004. Genetic drift outweighs balancing selection in shaping post-bottleneck major histocompatibility complex variation in New Zealand robins (Petroicidae). Molecular Ecology 13: 3709-3721.

MousadikA, PetitR 1996. High level of genetic differentiation for allelic richness among populations of the argan tree [Argania spinosa (L.) Skeels] endemic to Morocco. Theoretical and Applied Genetics 92: 832-839.

Nei M 1978. Estimation of average heterozygosity and genetic distance from a small number of individuals. Genetics 89: 583-590.

Oliver M, Telfer S, Piertney S 2009. Major histocompatibility complex (MHC) heterozygote superiority to natural multiparasite infections in the water vole (Arvicola terrestris). Proceedings of the Royal Society of London B: Biological Sciences 276: 1119-1128.

Palinauskas V, Valkiūnas G, Bolshakov CV, Bensch S 2008. Plasmodium relictum (lineage P-SGS1): effects on experimentally infected passerine birds. Experimental Parasitology 120: 372-380.

Paradis E 2010. pegas: an R package for population genetics with an integrated-modular approach. Bioinformatics 26: 419-420.

Paradis E, Claude J, Strimmer K 2004. APE: analyses of phylogenetics and evolution in R language. Bioinformatics 20: 289-290.

PatersonS, WilsonK,PembertonJ1998.Majorhistocompatibility complex variation associated with juvenile survival and parasite resistance in a large unmanaged ungulate population (Ovis aries L.). Proceedings of the National Academy of Sciences 95: 3714-3719.

Peakall R, Smouse PE 2006. GENALEX 6: genetic analysis in Excel. Population genetic software for teaching and research. Molecular Ecology Notes 6: 288-295.

Petit RJ, El MousadikA, Pons O 1998. Identifying populations for conservation on the basis of genetic markers. Conservation Biology 12: 844-855.

Piertney SB, Oliver MK 2006. The evolutionary ecology of the major histocompatibility complex. Heredity 96: 7-21.

R CoreTeam 2014. R:Alanguage and environment for statistical computing. R Foundation for Statistical Computing, Vienna, Austria. http://www.R-project.org/ (accessed 10 December 2015).

Radwan J, Biedrzycka A, Babik W 2010. Does reduced MHC diversity decrease viability of vertebrate populations? Biological Conservation 143: 537-544.

Radwan J, Zagalska-Neubauer M, CichoŃ M, Sendecka J, Kulma K, Gustafsson L, Babik W 2012. MHC diversity, malaria and lifetime reproductive success in collared flycatchers. Molecular Ecology 21: 2469-2479.

Raymond M, RoussetF 1995. Genepop(version 1.2): population genetics software for exact tests and ecumenicism. Journal 
of Heredity 86: 248-249.

Rice WR 1989. Analyzing tables of statistical tests. Evolution: 223-225.

Rothberg JM, Hinz W, Rearick TM, Schultz J, Mileski W, Davey M, Leamon JH, Johnson K, Milgrew MJ, Edwards M, Hoon J, Simons JF, Marran D, Myers JW, Davidson JF, Branting A, Nobile JR, Puc BP, Light D, Clark TA, Huber M, Branciforte JT, Stoner IB, Cawley SE, Lyons M, Fu Y, Homer N, Sedova M, Miao X, Reed B, Sabina J, Feierstein E, Schorn M, Alanjary M, Dimalanta E, Dressman D, Kasinskas R, Sokolsky T, Fidanza JA, Namsaraev E, McKernan KJ, Williams A, Roth GT, Bustillo J 2011. An integrated semiconductor device enabling non-optical genome sequencing. Nature 475: 348-352.

Rousset F 2008. genepop'007: a complete re-implementation of the genepop software for Windows and Linux. Molecular Ecology Resources 8: 103-106.

Sandberg M, Eriksson L, Jonsson J, Sjöström M, Wold S 1998. New chemical descriptors relevant for the design of biologically active peptides. Amultivariate characterization of 87 amino acids. Journal of Medicinal Chemistry 41: 2481-2491.

Schoener E, Banda M, Howe L, Castro I, Alley M 2014. Avian malaria in New Zealand. New Zealand Veterinary Journal 62: 189-198.

Schuelke M 2000. An economic method for the fluorescent labeling of PCR fragments. Nature Biotechnology 18: 233-234.

Schwensow N, Fietz J, Dausmann KH, Sommer S 2007. Neutral versus adaptive genetic variation in parasite resistance: importance of major histocompatibility complex supertypes in a free-ranging primate. Heredity 99:265-277.

Sepil I, Moghadam HK, Huchard E, Sheldon BC 2012. Characterization and 454 pyrosequencing of Major Histocompatibility Complex class I genes in the great tit reveal complexity in a passerine system. BMCEvolutionary Biology 12: 68.

Sepil I, Lachish S, Hinks AE, Sheldon BC 2013. Mhc supertypes confer both qualitative and quantitative resistance to avian malaria infections in a wild bird population. Proceedings of the Royal Society B Biological Sciences 280: 20130134.

Seutin G, White BN, Boag PT 1991. Preservation of avian blood and tissue samples for DNA analyses. Canadian Journal of Zoology 69: 82-90.

Siddle HV, Kreiss A, Eldridge MD, Noonan E, Clarke CJ, Pyecroft S, Woods GM, Belov K 2007. Transmission of a fatal clonal tumor by biting occurs due to depleted MHC diversity in a threatened carnivorous marsupial. Proceedings of the National Academy of Sciences 104: 16221-16226.

Siddle HV, Marzec J, Cheng Y, Jones M, Belov K 2010. MHC gene copy number variation in Tasmanian devils: implications for the spread of a contagious cancer. Proceedings of the Royal Society of London B: Biological Sciences 277: 2001-2006.

Slatkin M 1995. A measure of population subdivision based on microsatellite allele frequencies. Genetics 139: 457-462.

Sutton JT 2013. Major histocompatibility complex and microsatellite genetic diversity in bottlenecked populations of New Zealand passerines. PhD thesis, University of Otago, Dunedin.

Sutton JT, Robertson BC, Jamieson IG 2011. Dye shift: a neglected source of genotyping error in molecular ecology. Molecular Ecology Resources 11: 514-520.
Sutton JT, Robertson BC, Grueber CE, Stanton JL, Jamieson IG 2013. Characterization of MHC class II B polymorphism in bottlenecked New Zealand saddlebacks reveals low levels of genetic diversity. Immunogenetics 65: 619-633.

Sutton JT, Robertson BC, Jamieson IG 2015. MHC variation reflects the bottleneck histories of New Zealand passerines. Molecular Ecology 24: 362-373.

Thorne JM 2007. An experimental approach to translocation of the North Island saddleback (Philesturnus carunculatus rufusater) to Bushy Park Reserve, Wanganui. MSc Thesis, Massey University, Palmerston North.

Thursz MR, Kwiatkowski D, Allsopp CE, Greenwood BM, Thomas HC, Hill AV 1995. Association between an MHC class II allele and clearance of hepatitis B virus in the Gambia. New England Journal of Medicine 332: 1065-1069.

Tompkins DM, Gleeson DM 2006. Relationship between avian malaria distribution and an exotic invasive mosquito in New Zealand. Journal of the Royal Society of New Zealand 36: 51-62.

Trachtenberg E, Korber B, Sollars C, Kepler TB, Hraber PT, Hayes E, Funkhouser R, Fugate M, Theiler J, Hsu YS 2003. Advantage of rare HLA supertype in HIV disease progression. Nature Medicine 9: 928-935.

Valkiūnas G, Anwar AM, Atkinson CT, Greiner EC, Paperna I, Peirce MA2005. What distinguishes malaria parasites from other pigmented haemosporidians? Trends in Parasitology 21: $357-358$

van OosterhoutC, Hutchinson WF, Wills DPM, Shipley P2004. micro-checker: software for identifying and correcting genotyping errors in microsatellite data. MolecularEcology Notes 4: 535-538.

van Riper III C, van Riper SG, Goff ML, Laird M 1986. The epizootiology and ecological significance of malaria in Hawaiian land birds. Ecological Monographs 56: 327-344.

Walsh PS, Metzger DA, Higuchi R 1991. Chelex-100 as a medium for simple extraction of dna for pcr-based typing from forensic material. Biotechniques 10: 506-513.

Wegner KM, Kalbe M, Kurtz J, Reusch TB, Milinski M 2003. Parasite selection for immunogenetic optimality. Science 301: 1343-1343.

Westerdahl H, Waldenstrom J, Hansson B, Hasselquist D, von Schantz T, Bensch S 2005. Associations between malaria and $\mathrm{MHC}$ genes in a migratory songbird. Proceedings of the Royal Society B: Biological Sciences 272: 1511-1518.

Westerdahl H, Asghar M, Hasselquist D, Bensch S 2012. Quantitative disease resistance: to better understand parasite-mediated selection on major histocompatibility complex. Proceedings of the Royal Society of London B: Biological Sciences 279: 577-584.

Yuhki N, O'Brien SJ 1990. DNA variation of the mammalian major histocompatibility complex reflects genomic diversity and population history. Proceedings of the National Academy of Sciences 87: 836-840.

Zagalska-Neubauer M, Babik W, Stuglik M, Gustafsson L, Cichoń M, Radwan J 2010. 454 sequencing reveals extreme complexity of the class II Major Histocompatibility Complex in the collared flycatcher. BMC Evolutionary Biology 10: 395.

Editorial board member: Hannah Buckley

Received 13 August 2015; accepted 3 December 2015 


\section{Supplementary Material}

Additional supporting information may be found in the online version of this article:

Table S1: Microsatellite primers and fluorophore labels used to quantify neutral genetic variation in North Island saddlebacks (Philesturnus rufusater) from Mokoia Island.

The New Zealand Journal of Ecology provides online supporting information supplied by the authors where this may assist readers. Such materials are peer-reviewed and copy-edited but any issues relating to this information (other than missing files) should be addressed to the authors. 